\title{
Racism and gynecologic surgery: A time to act
}

\author{
The data suggest a deeper dive is required for understanding \\ how race factors into gyn surgery outcomes, and what we can do, \\ beyond recognition, to re-frame our approaches so that health equity \\ can be achieved
}

Cynthia Arvizo, MD, and Laveena Kondagari, MD

IN THIS ARTICLE

Hysterectomy

approach

and racism

this page

A conceptual re-framework page 34 lthough recent events have spurred much discourse regarding systemic racism, the issue of racism is old, very old. Unfortunately, our gynecologic surgery history is rooted in racism, with numerous documented procedures performed on enslaved women without their consent. Over the years, racism has continued to permeate gynecologic surgery in so far as access to quality care, patient outcomes, and inclusion in research. While racial disparities with regard to stage at diagnosis and survival of gynecologic malignancy has been documented, this discussion is outside the scope of this article.

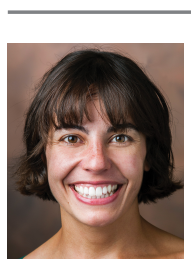

Dr. Arvizo is Director of Minimally Invasive Gynecologic Surgery, Jacobi Medical Center, and Assistant Professor, Albert Einstein College of Medicine, Bronx, New York.

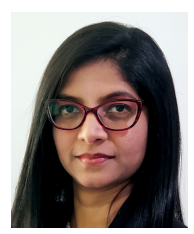

Dr. Kondagari is Director of Gynecologic Ultrasound Unit, Jacobi Medical Center, and Assistant Professor, Albert Einstein College of Medicine.

The authors report no financial relationships relevant to this article.

doi: 10.12788 /obgm.0075

\section{Racial disparities in gyn} surgery: The evidence

More data exist with regard to hysterectomy and racism than with any other gynecologic surgery. Most notably, a minimally invasive approach to hysterectomy is less likely to occur for minority women, even in universally insured patient populations and when controlling for factors predisposing patients to an abdominal approach.

\section{Minority women undergo MIS for hysterectomy less often}

Ranjit and colleagues assessed hysterectomy data between 2006 and 2010 from National TRICARE Prime and Prime Plus data to evaluate if racial differences existed in a universally insured population of US Armed Services members and their dependents. African American patients were significantly less likely than White patients to undergo a total vaginal hysterectomy (relative risk ratio [RRR], 0.63; 95\% confidence interval [CI], 0.58-0.69) or total laparoscopic hysterectomy (RRR, 0.65; 95\% CI, 0.60-0.71) compared with abdominal hysterectomy. Asian patients were also less likely to receive the vaginal (RRR, 0.71 ; 95\% CI, 0.60 0.84 ) or laparoscopic (RRR, 0.69 ; $95 \% \mathrm{CI}$, 0.58-0.83) approach to hysterectomy than White patients. ${ }^{1}$ These findings remained when controlled for surgery indication, suggesting that racial inequity was not attributed solely to preoperative patient factors. 
However, the authors could not control for specific patient factors such as body mass index and uterine weight.

Katon and colleagues reviewed data on patients who underwent hysterectomy for uterine fibroids at a Veterans Affairs hospital and found 99 excess abdominal hysterectomies were performed among Black women compared with White women. Despite controlling for predisposing factors related to abdominal surgery, facility, and geography (teaching hospital, higher volume hysterectomy), Black women were still less likely to undergo minimally invasive hysterectomy. ${ }^{2}$ The difference in approach between both groups remained largely unexplained. ${ }^{2}$

Pollack and colleagues reviewed hysterectomy data from Agency for Healthcare Research and Quality Healthcare Cost and Utilization Project State Inpatient Database and State Ambulatory Surgery Databases between 2010 and 2014 from Colorado, Florida, Maryland, New Jersey, and New York. They found that African American and Hispanic women were less likely to undergo vaginal (adjusted standardized prevalence ratio [aPR], 0.93; 95\% CI, 0.90-0.96 and aPR, 0.95; 95\% CI, 0.93-0.97, respectively) and laparoscopic hysterectomy (aPR, 0.90; 95\% CI, 0.87-0.94 and aPR, 0.95; 95\% CI, 0.92-0.98, respectively) than White women. Asian/ Pacific Islander women were less likely to undergo vaginal hysterectomy (aPR, 0.88; 95\% CI, 0.81-0.96). They also found that hospitals providing care to more racial/ethnic minority women performed more abdominal and fewer vaginal procedures compared with other hospitals. ${ }^{3}$

Sanei-Moghaddam and colleagues reviewed data from University of Pittsburgh Medical Center-affiliated hospitals and found that European-American women had 0.47 times lower odds of undergoing abdominal hysterectomy compared with ethnic/ race minority group women. Also, traditional Medicaid and Medicare enrollees had 2- to 4-times higher odds of having an abdominal hysterectomy compared with patients with commercial insurance. ${ }^{4}$ Evidently, insurance and payer status and hospital, along with race, were associated with abdominal hysterectomy.

Postop complications higher among Black women. One study of the National Surgical Quality Improvement Program 2015 hysterectomy database found that Black women were more likely to undergo open hysterectomy than White women despite controlling for patient factors associated with open hysterectomy, including uterine weight (adjusted odds ratio [aOR], 2.02; 95\% CI, 1.85-2.20). ${ }^{5}$ Black women also were more likely to develop both minor and major postoperative complications despite controlling for route of hysterectomy (major complications aOR, 1.56; 95\% CI, 1.25-1.95 and minor complications aOR, 1.27; 95\% CI, 1.11-1.47). Their study was limited by inability to control for surgeon volume and experience and hospital-specific factors. ${ }^{5}$

Hospital size and surgeon volume found to play a role in disparities. In an effort to address hospital and surgeon factors and racial disparities in minimally invasive hysterectomy, Mehta and colleagues evaluated an all payer system in Maryland. Black (reference White; aOR, 0.70; 95\% CI, 0.63-0.78) and Hispanic patients (aOR, 0.62; 95\% CI, 0.48-0.80) were less likely to undergo minimally invasive hysterectomy. Patients who had surgery at small- and medium-sized hospitals or by medium-volume surgeons (medium vs high volume: OR, 0.78; 95\% CI, 0.71-0.87) were also more likely to undergo open hysterectomy. ${ }^{6}$ The study authors suggest increased utilization of higher volume surgeons for referrals or to assist lower-volume surgeons as potential solutions to address racial disparities. ${ }^{6}$

\section{Surgical outcome disparities extend beyond hysterectomy route}

While the bulk of data with regard to gynecologic surgery and racism addresses minimally invasive approach to treatment of fibroids and hysterectomy, limited data regarding ectopic pregnancy and adnexal surgery reveal similar findings. Hsu and colleagues reported that Black (adjusted risk ratio [aRR], 0.76; 95\% CI, 0.69-0.85) and Hispanic (aRR, 0.80; 95\% CI, 0.66-0.96) women
FAST

TRACK

Data suggest racial disparities in hysterectomy approach, postoperative complications, and tubal-sparing procedures for ectopic pregnancy 
TABLE 1 Examples of manifestations of racism at each level

\begin{tabular}{l|l|l}
\multicolumn{1}{c}{ Systemic } & \multicolumn{1}{c}{ Personally mediated } & Internalized \\
\hline - Access to care & - Implicit bias & - Imposter syndrome \\
- Existing laws & - Dehumanization & - Lack of shared decision making \\
- Insurance status & - Attribution of race as a biologic factor & - Lack of patient autonomy \\
- Education & - Paternalism & \\
- Lack of research & & \\
- Lack of representation & & \\
- Medical facilities & & \\
\hline
\end{tabular}

FAST

TRACK

Employing a

conceptual

framework that

considers the

different levels

of racism can be

a starting point

for addressing

racial disparities

in gynecologic

surgery treated surgically for ectopic pregnancy were less likely to undergo tubal-sparing procedures than White women. ${ }^{7}$ Their study did not control for human chorionic gonadotropin levels, ectopic size, or comorbidities as measured by the Elixhauser Comorbidity Index.

The data regarding gynecologic surgery and racial inequity are sparse but manifest differences that are unexplained entirely by patient payer status and individual patient factors. Studies do confirm hospital and surgeon characteristics play a part in provision of minimally invasive hysterectomy.

\section{Forming a conceptual re-framework to achieve health equity}

The centuries-long impact of racism on our field, and more specifically on gynecologic surgery, will take time and a conscious effort to overcome. In 2001, the Institute of Medicine outlined 6 domains for improvement, amongst them equitable care-"ensuring quality of care does not vary because of characteristics." ${ }^{\prime 8}$ As highlighted above, some aspects of gynecologic surgery have proven to be inequitable, specifically in the provision of minimally invasive hysterectomy and treatment of ectopic pregnancy in Black women. The lack of studies on racism and gynecologic surgery as it pertains to other benign gynecologic conditions highlights the need for more research and measures that target each level of racism and, ultimately, achieve health equity.

Priority \#1: Support and funding. In 2016, the Institute for Healthcare Improvement (IHI) published a white paper describing a framework to bring about health equity. First and foremost, institutions and individuals must prioritize health equity by obtaining leadership support and adequate funding. ${ }^{9}$ In August 2020, several leading obstetrics and gynecology organizations published a joint statement highlighting their initial plan of action to address racism and provide equitable care. ${ }^{10}$ As leading professional organizations prioritize equity, we can hope institutions and departments continue to do so as well.

Priority \#2: Measuring the extent of the problem. Once adequate support and funding is established, the IHI recommends ${ }^{9}$ :

- establishing structures and processes with an overseeing committee and dedicated budget

- deploying strategies with comprehensive data collection and pertinent metrics.

\section{Applying the levels of racism to a new framework}

Given the numerous untouched areas of research and components contributing to racial disparities in gynecologic surgery, determining a starting point can prove overwhelming. We suggest employing a conceptual framework that considers the different levels of racism (TABLE 1).

Three different levels of racism have been described previously:

- systemic/institutionalized,

- personally mediated

- internalized. ${ }^{11,12}$

Systemic racism refers to differential access to services and goods in society and power within society, for example housing, 
TABLE 2 Example processes to improve access to minimally invasive surgery

\begin{tabular}{|c|c|c|c|}
\hline Department & Hospital & Community organization & Professional organizations \\
\hline $\begin{array}{l}\text { - Quality improvement projects } \\
\text { based on institution-specific } \\
\text { disparity data } \\
\text { - Shared decision-making guides } \\
\text { - Referral system } \\
\text { - Provision of equipment } \\
\text { - Departmental guidelines for care } \\
\text { - Models for surgical coaching }\end{array}$ & $\begin{array}{l}\text { - Leadership buy-in } \\
\text { - Sustainable funding } \\
\text { - Recruiting and } \\
\text { retaining minority staff } \\
\text { - Staff professional } \\
\text { development } \\
\text { - Policies increasing } \\
\text { access to care }\end{array}$ & $\begin{array}{l}\text { Patient and community } \\
\text { education regarding } \\
\text { options for care } \\
\text { - Teaming up with } \\
\text { community health care } \\
\text { workers to improve early } \\
\text { detection, management, } \\
\text { and prevention of diseases }\end{array}$ & $\begin{array}{l}\text { - Advocacy for better local, } \\
\text { state, and national policies } \\
\text { on health equity } \\
\text { - Establishment of standard } \\
\text { of care guidelines } \\
\text { - Surgeon accreditation }\end{array}$ \\
\hline
\end{tabular}

education, medical care, and voting and representation. ${ }^{12}$ Systemic racism is arguably the overarching form of racism. The studies by Mehta and colleagues and Pollack et al specifically highlight a lack of adequate access to minimally invasive hysterectomy and a subsequent increase in complication rates in minority race groups. ${ }^{3,13}$ Access to care is only one example of systemic racism that requires action at multiple levels by professional organizations, hospitals, community organizations, and individual departments with multiple targeted solutions (TABLE 2).

Mediated racism. The second form of racism is personally mediated racism, in other words discrimination and prejudice formed by preconceived notions of a person based on their race. ${ }^{12}$ In the joint statement published by the leading obstetrics and gynecology organizations in August 2020, a recognition of race as a social construct without the biological weight we have long afforded it was made explicit. This realization can be applied in the day-to-day categorization of patients and, most notably, the formation of a diagnosis and treatment plan.

A concrete example of potentially biased treatment is illustrated when limiting management options to the "unreliable" patient. Exposure to stereotypes and misinformation can develop into implicit bias and subsequently make the most intelligent, compassionate provider show behavior with microaggressions. This subtle behavior can play a major role in patient-provider communication and in turn affect care satisfaction, provider trust, and shared decision making. ${ }^{14}$
The Implicit bias Association Test or MPathicVR virtual human simulations can be used to identify provider-specific implicit bias. ${ }^{14,15}$

Internalized racism. Lastly, internalized racism refers to the individual's acceptance of negative messages regarding their own abilities and worth, ${ }^{12}$ which is seen commonly in imposter syndrome. Imposter syndrome, which is a failure to internalize one's own successes and persistent fear of being discovered as a fraud, a condition which has been more commonly seen in ethnic minority groups. ${ }^{16} \mathrm{~A}$ patient's internalized racism can manifest as self-devaluation and helplessness which may make a patient less likely to question their treatment. ${ }^{12,17}$ Moreover, some evidence exists indicating that patients with diabetes identified physician discrimination and internalized racism as factors impeding shared decision making. ${ }^{18}$

\section{The next steps first require recognition}

Racial inequity has long infiltrated our medical field and the discussion surrounding the effects of racism on our patients and providers, and research, is long overdue. Although research continues to emerge regarding race inequity and gynecologic surgery, much remains to be done. In recognizing the levels of racism and the roles they play in our provision of good, equitable, patient-centered care, we-as individuals, departments, and organizations-can combat racism and strive for health equity.
FAST

TRACK

Targeting multiple solutions against systemic racism requires action at multiple levels by professional organizations, hospitals, community organizations, and individual departments 


\section{Inequities in MEDICINE}

CONTINUED FROM PAGE 35

\section{References}

1. Ranjit A, Sharma M, Romano A, et al. Does universal insurance mitigate racial differences in minimally invasive hysterectomy? J Minim Invasive Gynecol. 2017;24:790-796.

2. Katon JG, Bossick AS, Doll KM, et al. Contributors to racial disparities in minimally invasive hysterectomy in the US Department of Veterans Affairs. Med Care. 2019;57:930-936.

3. Pollack LM, Olsen MA, Gehlert SJ, et al. Racial/ethnic disparities/differences in hysterectomy route in women likely eligible for minimally invasive surgery. J Minim Invasive Gynecol. 2020;27:1167-1177.e2.

4. Sanei-Moghaddam A, Kang C, Edwards RP, et al. Racial and socioeconomic disparities in hysterectomy route for benign conditions. J Racial Ethn Health Disparities. 2018;5:758-765.

5. Alexander AL, Strohl AE, Rieder S, et al. Examining disparities in route of surgery and postoperative complications in black race and hysterectomy. Obstet Gynecol. 2019;133:6-12.

6. Mehta A, Xu T, Hutfless S, et al. Patient, surgeon, and hospital disparities associated with benign hysterectomy approach and perioperative complications. Am J Obstet Gynecol. 2017;216:497.el-497.e10.

7. Hsu JY, Chen L, Gumer AR, et al. Disparities in the management of ectopic pregnancy. Am J Obstet Gynecol. 2017;217:49. el-49.e10.

8. Institute of Medicine Committee on Quality of Health Care in America. Crossing the Quality Chasm: A New Health System for the 21st Century. Washington DC: National Academies Press; 2001.

9. Wyatt R, Laderman M, Botwinick L, et al. Achieving Health Equity: A Guide for Health Care Organizations. Cambridge, MA: Institute for Healthcare Improvement; 2016.

10. Joint Statement: Collective Action Addressing Racism. AAGL web site. https://www.aagl.org/aaglnews/joint-statement -collective-action-addressing-racism/. Released August 27 , 2020. Accessed January 22, 2021.

11. Paradies Y, Ben J, Denson N, et al. Racism as a determinant of health: a systematic review and meta-analysis. PLoS One. 2015;10:e0138511.

12. Jones CP. Levels of racism: a theoretic framework and a gardener's tale. Am J Public Health. 2000;90:1212-1215.

13. Mehta A, Xu T, Hutfless S, et al. Patient, surgeon, and hospital disparities associated with benign hysterectomy approach and perioperative complications. Am J Obstet Gynecol. 2017;216:497.el-497.e10.

14. Hagiwara N, Elston Lafata J, Mezuk B, et al. Detecting implicit racial bias in provider communication behaviors to reduce disparities in healthcare: challenges, solutions, and future directions for provider communication training. Patient Educ Couns. 2019;102:1738-1743.

15. Kron FW, Detters MD, Scerbo MW, et al. Using a computer simulation for teaching communication skills: A blinded multisite mixed methods randomized controlled trial. Patient Educ Couns. 2017;100:748-759.

16. Bravata DM, Watts SA, Keefer AL, et al. Prevalence, predictors, and treatment of impostor syndrome: a systematic review. J Gen Intern Med. 2020;35:1252.

17. Peek ME, Odoms-Young A, Quinn MT, et al. Racism in healthcare: its relationship to shared decision-making and health disparities: a response to Bradby. Soc Sci Med. 2010;71:13.

18. Peek MA, Odoms-Young A, Quinn MT, et al. Race and shared decision-making: perspectives of African-Americans with diabetes. Soc Sci Med. 2010;71:1-9. 